\title{
ION ETCHING EFFECTS OCCURRING IN SECONDARY ION MASS SPECTROMETRY DEPTH PROFILING OF InGaAs/InP AND InGaAs/AlAs/InP MBE GROWN HETEROSTRUCTURES
}

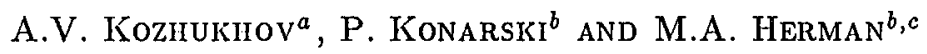 \\ ${ }^{a}$ Institute of Semiconductors Physics, Russian Academy of Sciences \\ 13'Lauvrentjeva Ave., 630090 Novosibirsk, Russia \\ ${ }^{b}$ Institute of Vacuum Technology, Długa 44/50, 00-241 Warsaw, Poland \\ 'Institute of Physics, Polish Academy of Sciences \\ Al. Lotników 32/46, 02-668 Warszawa, Poland
}

Depth profiling analysis of $\operatorname{In}_{x} \mathrm{Ga}_{1-x}$ As heterolayers grown by MBE on Fe doped In $\mathrm{P}(100)$ substrates was performed in the SAJW-02 secondary ion mass spectrometry analyser equipped with $4.5 \mathrm{keV} \mathrm{O}+$ ion source and a specially designed sample manipulator enabling depth profiling in the standard as well as in so-called Zalar rotation operation modes. The fairly high energy of the primary ion beam required for sputtering in secondary ion mass spectrometry measurements causes changes in surface topography, usually of different origin. Depth resolution parameters and roughness formation monitored by scanning electron microscopy were analysed for a set of samples with composition $x$ changing in the range 0.33 to 0.60 . The results were compared with the same data for a layer of $x=0.53$ (best lattice-matched to InP) grown on the top of a three monolayer thick AlAs film deposited previously on the InP substrate. Improvement in the depth profile resolution was revealed for the structure with an AlAs layer indicating sharper interface transition. Moreover, sample rotation applied for this structure improves further the depth profiling resolution. Thus, we showed for the first time that a very thin AlAs layer grown by MBE between the InP substrate and the $\operatorname{In}_{0.53} \mathrm{Ga}_{0.47}$ As improves considerably the heterointerface properties and that Zalar rotation applied for depth profiling of the investigated material system diminishes further the negative effects of ion etching on depth resolution in secondary ion mass spectrometry analysis.

PACS numbers: 68.35.Bs, 68.55.Jk 


\section{Introduction}

The standard procedure of secondary ion mass spectrometry (SIMS) depth profile analysis of semiconductor heterostructures is based on sequential removal of atomic layers and the subsequent analysis of their elemental concentration by monitoring the secondary ion emission. One of the major problems inherent in SIMS depth profile analysis is surface roughening which occurs during the erosion of surface caused by ion beam bombardment [1].

The fairly high energy of the primary ion beam $(\approx 5 \mathrm{keV})$ causes changes in the properties of a definite layer near the surface of the analysed structure due to ion mixing, ion-induced diffusion or saturation of the bombarding ions [2].

Beside these effects occurring beneath the surface, changes in surface topography may also appear. These changes may be connected, for example, with non-uniform current density of the applied rastered ion beam, with the statistical character of the sputtering effect, or with the angular anisotropy of the sputtering coefficient.

One way of a voiding surface morphology development during ion sputtering is ion bombardment at glancing incidence angles. Also two-directional bombardment was proposed, as well as continuous sample rotation, originally applied by Zalar for Auger electron spectroscopy depth profiling [3]. The general effect of sample rotation as applied to SIMS depth profiling is the improvement of depth resolution and its independence of the sputtered crater depth $[1,4]$.

In this paper we present and shortly discuss the experimental results concerning the Gaussian depth resolution parameter $\Delta z$ (defined as the sputtered depth range related to the change of the secondary ion's current from $84 \%$ to $16 \%$ during passing an interface by the ion-etching front) and roughness formation phenomenon (monitored by scanning electron microscopy - SEM) measured at SIMS depth profiling for a set of heterostructures of $\operatorname{In}_{x} \mathrm{Ga}_{1-x} \mathrm{As} / \mathrm{InP}(x=0.33,0.53$, $0.60)$ grown by solid source MBE [5]. The results obtained for the heterostructure $\mathrm{In}_{0.53} \mathrm{Ga}_{0.47} \mathrm{As} / \mathrm{InP}$ (best lattice matched) were compared with the same data measured for the $\operatorname{In}_{0.53} \mathrm{Ga}_{0.47} \mathrm{As} / \mathrm{AlAs} / \mathrm{InP}$ heterostructure, with a very thin $(\approx 3$ monolayers $(\mathrm{ML}))$ AlAs layer deposited between the InP substrate and the $\mathrm{In}_{0.53} \mathrm{Ga}_{0.47} \mathrm{As}$ heterolayer.

\section{Experimental}

The MBE growth processes of the investigated InGaAs/InP and InGaAs/ $\mathrm{AlAs} / \mathrm{InP}$ heterostructures were performed in the MBE system "Katun - B" (designed and manufactured by the Institute of Semiconductor Physics, RAS, Novosibirsk, Russia). In situ control of the growth processes was carried out by reflection high energy electron diffraction (RHEED) and by quadrupole mass spectrometry (QMS). The InGaAs films were grown on Fe doped InP(100) epi-ready substrates. Before the MBE growth, oxide desorption from the substrate surface was caused by heating the substrate under an $\mathrm{As}_{4}$ flux at temperatures $510-530^{\circ} \mathrm{C}$. Simultaneously, changes in the RHEED patterns from the substrate surface, were recorded. When the indium stabilised $4 \times 2$ surface reconstruction occurred the InGaAs films of different composition were grown by MBE. 
In the case of the growth of InGaAs/AlAs/InP structures, the indium stabilised InP surface heated under an $\mathrm{As}_{4}$ flux was first covered by a very thin (3 atomic MLs) Al layer, which resulted in the AlAs/InP heterointerface. Subsequently, the $\mathrm{In}_{0.53} \mathrm{Ga}_{0.47} \mathrm{As}$ layer was grown in a standard way.

The depth profile analyses of the investigated heterostructures were performed on SIMS analyser SAJW-02 designed and manufactured in the Institute of Vacuum Technology, Warsaw. The base parameters of this analyser are: primary ion beam current $100 \mathrm{nA}$, energy $4.5 \mathrm{keV}$, beam diameter $100 \mu \mathrm{m}$, angle of incidence $45^{\circ}$, bombarding ions $\mathrm{O}_{2}^{+}$or $\mathrm{Ar}^{+}$.

In this study we apply the sample rotation technique [4] using special UHV sample rotation manipulator [6] which allows precise positioning and sample rotation around the surface normal. Sample rotation at $1.5 \mathrm{rpm}$ rate was digitally controlled and thus monitoring of the secondary ion currents at a chosen azimuth of the rotating sample was possible.

\section{Results concerning ion etching effects occurring in SIMS depth profiling}

The SIMS depth profiling experimental results showing the ion etching effects on depth resolution $\Delta z$ are shown in Fig. 1 and Fig. 2. Figure 1 shows the depth profiles of the $\operatorname{In}_{x} \mathrm{Ga}_{1-x} \mathrm{As} / \mathrm{InP}(x=0.6,0.53,0.33)$ heterointerfaces for the ${ }^{71} \mathrm{Ga}^{+}$secondary ions of gallium. One may easily recognise that the increase in In content in the epilayer from $x=0.33$ to $x=0.6$ causes the increase in $\Delta z_{\mathrm{Ga}}$ from $29 \mathrm{~nm}$ to $97 \mathrm{~nm}$, respectively (this means a decrease in depth profile resolution). Figure 2 shows a set of SEM micrographs indicating the surface

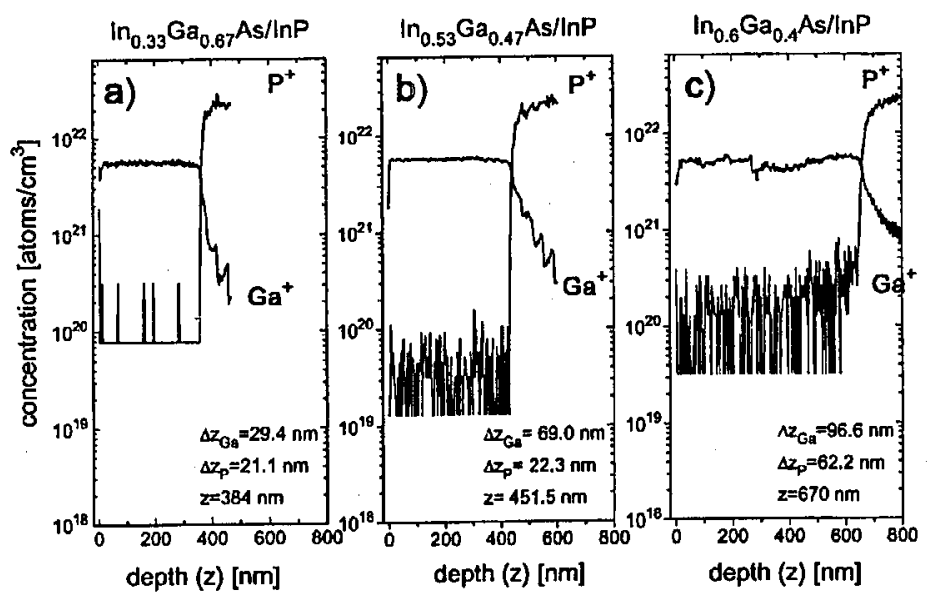

Fig. 1. SIMS depth profile analysis of three MBE grown $\operatorname{In}_{x} \mathrm{Ga}_{1-x} \mathrm{As} / \mathrm{InP}$ heterostructures with $x=0.33(\mathrm{a}), x=0.53$ (b) and $x=0.60$ (c). Depth resolution parameters " $\Delta z$ " measured at the interface depth " $z$ ", are specified for phosphorus and gallium. Depth calibration was done after profilometer ( $\alpha$-step Tencor 100) testing of the eroded craters. 

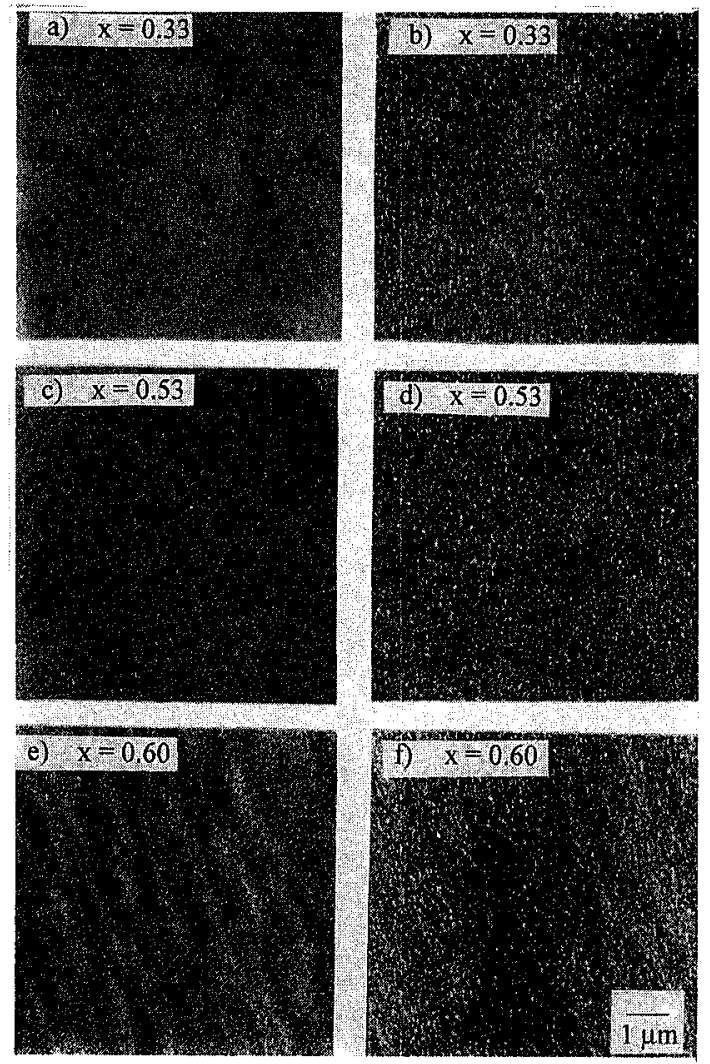

Fig. 2. Scanning electron microscopy (SEM) micrographs of virgin surfaces ((a), (c), (e)) and ion eroded crater bottoms ((b), (d), (f)) in three analysed $\operatorname{In}_{x} \mathrm{Ga}_{1-x} \mathrm{As} / \mathrm{InP}$ structures. All craters were eroded with $4.5 \mathrm{keV} \mathrm{O} \mathrm{O}_{2}^{+}$ions with incidence angle $45^{\circ}$ and in standard SIMS mode i.e. with constant azimuth angle. Composition parameter " $x$ " is specified. Magnification of all the micrographs is the same.

roughness of the bottom of the ion-etched crater, describing that the increase in ion etching-caused surface roughness causes the decrease in depth resolution. Figure 3 presents a comparison of the depth resolution data between the $\mathrm{In}_{0.53} \mathrm{Ga}_{0.47} \mathrm{As} / \mathrm{InP}$ and $\mathrm{In}_{0.53} \mathrm{Ga}_{0.47} \mathrm{As} / \mathrm{AlAs} / \mathrm{InP}$ heterostructures. The insets show also the positive effect of the sample rotation (Zalar rotation) on the ion etching-caused roughening phenomenon. One may easily recognise that the effect of the very thin (3 atomic MLs) AlAs layer leads to diminished ion etching-caused surface roughness and thus, to considerable increase in depth resolution at SIMS depth profile analysis.

\section{Discussion}

The roughness formation at the initial stage of the MBE growth of InGaAs on InP is closely related to the lattice mismatch between the epilayer and the substrate. If the growth is proceeding in a layer-by-layer 2D mode (Frank- 


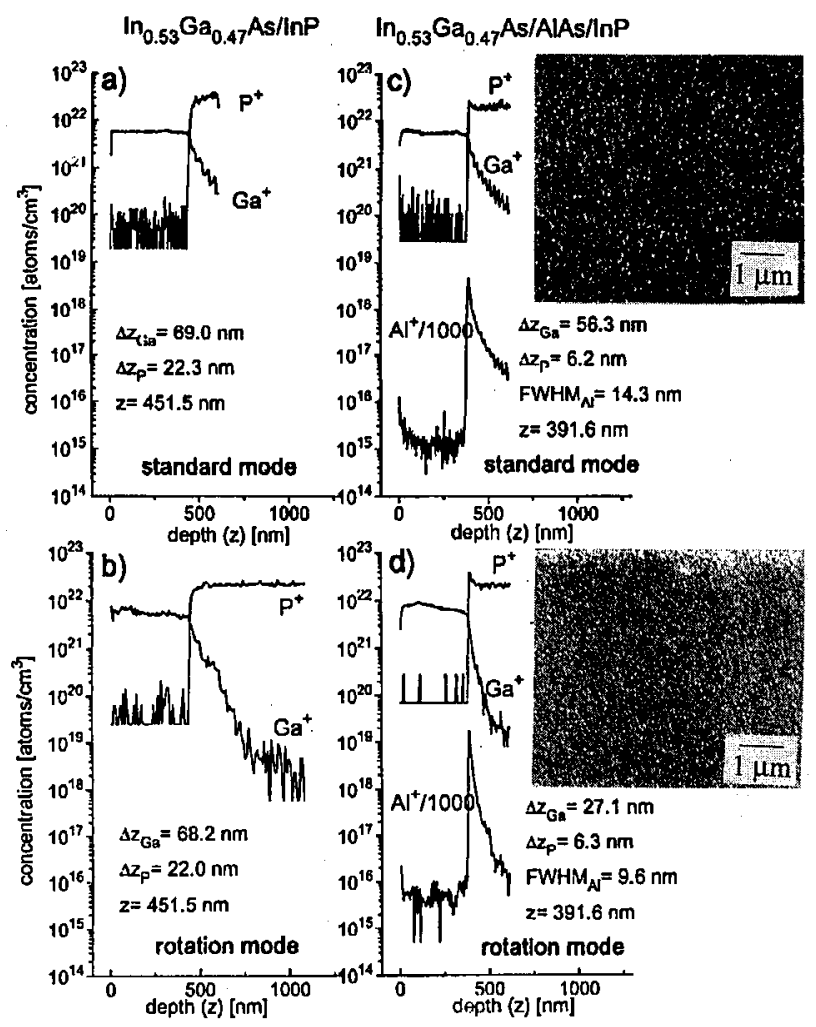

Fig. 3. Comparison of two structures $\operatorname{In}_{0.53} \mathrm{Ga}_{0.47} \mathrm{As} / \operatorname{InP}((\mathrm{a}), \quad(\mathrm{b}))$ and $\mathrm{In}_{0.53} \mathrm{Ga}_{0.47} \mathrm{As} / \mathrm{AlAs} / \mathrm{InP}((\mathrm{c}),(\mathrm{d}))$ analysed by SIMS in two operational modes: standard and rotation. Depth resolution parameters " $\Delta z$ " are given for $P$ and $G$ and FWHM parameter is specified for Al. In the insets ((c), (d)) the SEM micrographs of the eroded crater bottoms (InP) are shown for the $\mathrm{In}_{0.53} \mathrm{Ga}_{0.47} \mathrm{As} / \mathrm{AlAs} / \mathrm{InP}$ structure analysed in standard (c) and rotation (d) mode.

van der Merwe mode) the surface roughness is negligible. Consequently also the ion etching-caused roughening phenomenon is considerably decreased, thus, the depth resolution in SIMS depth profile analysis is accordingly increased. This effect is demonstrated by Fig. 1 and Fig. 2.

Figure 3 shows how Al deposition (creation of a very thin AlAs heterolayer) on the InP substrate diminishes the roughening phenomenon, thus, increases the depth resolution in SIMS depth profile analysis. This effect can be understood in two different ways. The first, taking into consideration only lattice mismatch in the heterostructure, assumes that an InAs near-surface layer (nearly $1 \mathrm{~nm}$ thick) is created in the InP substrate crystal during high temperature treatment under $\mathrm{As}_{4}$ flux (for desorbing the oxide from the epi-ready substrate surface). The mismatch between InAs and InP is equal to $7 \%$, which causes mechanical stress relaxed by creation of misfit dislocations which leads finally to a 3D mode of the growth of 
$\mathrm{In}_{0.53} \mathrm{Ga}_{0.47} \mathrm{As}$ epilayer (the interface is already atomically rough before ion etching of the SIMS analysis starts). However, addition of $\mathrm{Al}$ can reduce the $7 \%$ mismatch by formation of an AlAs layer much better lattice matched to the InP substrate. Thus, the interface between the $\mathrm{In}_{0.53} \mathrm{Ga}_{0.47} \mathrm{As} / \mathrm{AlAs}$ structure and InP is much smoother before starting the SIMS profiling procedure.

The second way takes into consideration $\mathrm{Al}$ induced In outdiffusion from the In-enriched near-surface layer of the InP substrate [7]. Such In-enriched substrate surface is usually not uniform, exhibiting a rough topography. Thus, a growth on such a rough surface leads to 3D mode of the MBE process. The topography of the substrate near-surface layer can be significantly improved prior to the growth of the InGaAs layer by deposition a thin Al layer on it. Reflection mass spectrometric investigations have evidenced [7] that Al deposition causes In desorption from the hot InP surface. As a result, the In-enriched near-surface layer of the InP substrate becomes now In-depleted, which reduces the roughness of the substrate surface. Thus three monolayers Al deposit can compensate the In-enriched layer forming instead of InAs an InAlAs layer. On the basis of the experimental results of Ref. [7], we believe that the second explanation is more realistic.

\section{References}

[1] P. Konarski, M.A. Herman, A.V. Kozhukhov, V.I. Obodnikov, Thin Solid Films 267, 114 (1995).

[2] Ion Bombardment Modification of Surfaces: Fundamentals and Applications, Eds. O. Auciello, R. Kelly, Elsevier, Amsterdam 1984.

[3] A. Zalar, Thin Solid Films 124, 223 (1985).

[4] E.H. Cirlin, J. Vajo, T.C. Hasenberg, R.J. Hauenstein, J. Vac. Sci. Technol. A 8, 4101 (1990).

[5] M.A. Herman, H. Sitter, Molecular Beam Epitaxy - Fundamentals and Current Status, 2nd ed., Springer, Berlin 1996.

[6] P. Konarski, Rev. Sci. Instrum. 66, 4713 (1995).

[7] A.V. Kozhukhov, M.A. Revenko, A.A. Fedorov, P. Konarski, M.A. Herman, Thin Solid Films, in press. 